\title{
The Role of Uncontrolled Eating and Screen Time in the Link of Attention Deficit Hyperactivity Disorder with Weight in Late Childhood
}

\author{
Joung-Sook Ahn, Seongho Min, and Min-Hyuk Kim ${ }^{凶}$ \\ Department of Psychiatry, Yonsei University, Wonju College of Medicine, Wonju, Republic of Korea
}

Objective The aim of this study was to examine the mediating roles of uncontrolled eating and sedentary behaviours in the link of attention deficit hyperactivity disorder (ADHD) and weight.

Methods A total of 352 children in fifth and sixth grade participated in the present study by completing the self-rated Three-Factor Eating Questionnaire and Children of Alcoholics Screening Test during regular classes. An additional questionnaire completed by their parents provided information about the children's ADHD and emotional symptoms, sedentary behaviour based on screen time, and parental variables. The questionnaires were surveyed within one week after their schools' annual physical check-up.

Results Hierarchical regression analyses revealed that uncontrolled eating was complete mediator in association between ADHD symptoms and body mass index (BMI) for boys, incomplete mediator for girls. However, screen time had no significant effect on the ADHD symptoms-BMI link for both gender.

Conclusion The findings of this study suggest that gender specific intervention programs may be need to help eating behaviour in children with ADHD and overweight. Psychiatry Investig 2017;14(6):808-816

Key Words Attention deficit hyperactivity disorder, Mediator, Uncontrolled eating, Screen time, Weight.

\section{INTRODUCTION}

Childhood overweight and obesity have become the global health issue of this century. For school-aged children and adolescents, "overweight" is defined by the World Health Organization growth reference as above one standard deviation body mass index (BMI) for age and gender and "obesity" as two standard deviations BMI. ${ }^{1}$ However, childhood overweight and obesity are considered equally associated with a high risk of being an obese adult. Therefore, in this paper, we consider overweight and obesity as the same condition and unify them as overweight. Despite various interventions focusing on dietary and physical activity patterns, the preva-

Received: January 5, 2017 Revised: May 17, 2017

Accepted: August 13, 2017 Available online: October 12, 2017

$\triangle$ Correspondence: Min-Hyuk Kim, MD

Department of Psychiatry, Yonsei University, Wonju College of Medicine, 20 Ilsan-ro, Wonju 26426, Republic of Korea

Tel: +82-33-741-1260, Fax: +82-33-741-0532, E-mail: mhkim09@yonsei.ac.kr

(a) This is an Open Access article distributed under the terms of the Creative Commons Attribution Non-Commercial License (http://creativecommons.org/licenses/by$\mathrm{nc} / 4.0$ ) which permits unrestricted non-commercial use, distribution, and reproduction in any medium, provided the original work is properly cited. lence of overweight in childhood has increased rapidly. ${ }^{1}$

A recent meta-analysis assessing the relationship between attention deficit hyperactivity disorder (ADHD) and childhood overweight showed that the pooled prevalence of overweight was greater by about $40 \%$ in children with ADHD than with children without ADHD. ${ }^{2}$ Although hyperactivity would seem to increase daily energy expenditure, a possible explanation for the comorbidity is that obesity genes in the pathways of dopaminergic circuitry have been associated with ADHD. ${ }^{3}$ A study using functional magnetic resonance imaging (fMRI) has identified inter-relationships among ADHD, obesity, and abnormal eating behaviour. Their shared neurobehavioral circuits are related to reward processing, response inhibition, and emotional processing and regulation. ${ }^{4}$ Thus, a dysregulated eating pattern through ADHD symptoms of impulsivity and inattention leading to weight gain is a possible explanation for the linkage. ${ }^{5,6}$ Intuitively, it would make sense that impulsive children with ADHD are less able to control their eating behaviour (uncontrolled eating) and are more likely to give in to the temptation of high-calorie food. ${ }^{7}$ Besides inattention, another core symptom of ADHD may be limited aware- 
ness of hunger and satiety. ${ }^{8}$

For the development of overweight in school-aged children, sedentary behaviour has been identified as an important risk factor, regardless of physical activity levels. ${ }^{9}$ Indeed, children with ADHD have been found to spend more time on the computer, playing video games, and watching TV, providing another explanation for an ADHD-overweight link..$^{10,11}$

In spite of numerous studies, there is still no consensus in hand about the association between ADHD and overweight, and several studies have reported no link between them. ${ }^{11,12}$ A plausible explanation of this discrepancy is the variety of confounders and wide range of age groups across studies, as suggested by Cortese et al. ${ }^{2}$ It has been reported that a number of variables as well as dietary patterns and physical activities have acted as confounders, such as parental BMI, parental smoking, low socioeconomic status (SES), and psychiatric comorbidity. Among psychiatric comorbidity, emotional disorder is a notable condition, given its high coexistence with both overweight and ADHD. ${ }^{13}$ In addition to confounders, the role of gender in the connection is also mixed. ${ }^{10,14,15}$ Some studies reported a significant gender effect, ${ }^{14,15}$ but others failed to find one. ${ }^{10}$

Taking all information into account, we assumed that if there is a link between ADHD and weight, it would preside over a complex path including mediators such as uncontrolled eating, sedentary behaviour, and various confounders. The aims of our study are, accordingly: 1) to clarify the role of uncontrolled eating and sedentary behaviour as mediators of the link between ADHD and weight and 2) to identify the gender difference in their relationship.

\section{METHODS}

\section{Participants and procedures}

The research was approved by the Ethics Committee of the Wonju College of Medicine, Yonsei University (YWMR-145-058). Informed consent was obtained from all participants. The study was then presented to three elementary schools, all of which are located in a city of 3 hundred thousand in population. We selected students in their late childhood, which is an important period in the development of eating attitudes and behaviours. ${ }^{16}$ A total of 352 children in the fifth and sixth grade participated in the present study by completing the selfrated Three-Factor Eating Questionnaire (TFEQ) and the Children of Alcoholics Screening Test (CAST) during regular classes. The teacher and a research assistant were present during the administration of the questionnaire to ensure independent and confidential responding and to provide assistance when necessary. An additional questionnaire completed by their parents provided information about the children's ADHD and emotional symptoms, sedentary behaviour based on screen time, and parental variables. The questionnaires were surveyed within one week after their schools' annual physical check-up in April or May. Children attending classes for the handicapped or mentally retarded were excluded from the study. Most of the non-responders were absent from the respective schools on the day the questionnaires were administered. Only 25 questionnaires were discarded because they were incomplete or inappropriately filled in. A total of 327 (157 from boys and 170 from girls) subjects were included in the data analysis.

\section{Measures}

\section{ADHD symptoms and emotional symptoms of the} Strengths and Difficulties Questionnaire (SDQ)

The parent-rated SDQ is composed of 25 items that can be used for screening psychopathology in children aged 7 to 14 years. ${ }^{17}$ For four difficulties domains, we selected the Hyperactivity-Inattention Scale (SDQ-HI) as an independent variable and the Emotional Symptoms Scale (SDQ-ES) as a confounder. The five items of the SDQ-HI assess three key symptoms for a DSM-IV diagnosis of ADHD. The five items of the SDQES evaluate somatic complaint, worry, unhappiness, nervousness, and fear symptoms. Each item of two scales was coded on a 3-point Likert scale. Therefore, scale scores ranged from 0 to 10 . One of the authors developed the Korean version of the SDQ. The psychometric property was acceptable with Cronbach's alpha 0.80 for the SDQ-HI and 0.61 for the SDQES. The SDQ-HI score $\geq 8$ point is pathological, 7 point is atrisk, and SDQ-ES $\geq 7$ point pathological, 6 point at-risk according to Korean clinical data. ${ }^{18}$

\section{Weight status}

Children's height and weight were measured by school nurses using a standardized protocol proposed by the regulation of the School Health Law. On the basis of the Korean reference data for children, the BMI calculated was transformed into an age- and gender-specific standard deviation score (BMI Z-score). Further, BMI cut-off points established by the Korea Centres for Disease Control and Prevention and Korean Paediatric Society in 2007 were used to define overweight, which corresponds to an adult BMI of $\geq 25.0 \mathrm{~kg} / \mathrm{m}^{2}{ }^{19}$

\section{Uncontrolled eating}

Eating behaviour was assessed by using a validated Korean version of the TFEQ, a self-reported scale designed to assess three cognitive and behavioural traits of eating: disinhibition, cognitive restraint, and hunger. The domain of disinhibition measures a loss of control in food intake by various external and internal stimuli that indicates uncontrolled eating. ${ }^{20}$ In 
the development process of the Korean version, wordings of some items were modified, and a few items were removed because differences in cultures and customs made it difficult to convey the original meaning. A 4-point Likert scale was adapted instead of the dichotomous rating of the original TFEQ. The 'true or false' format tends to place the participants in a situation in which neither choice is valid and to make them respond in socially desirable ways. ${ }^{21}$ As the disinhibition domain of the Korean version of TFEQ consisted of 14 items and was given a score between 1 and 4 for each item, scores ranged from 14 to 56, with a higher score being indicative of greater uncontrolled eating. A validation study replicated the factor structure of the original one and showed satisfying results with Cronbach's alpha 0.88 for the disinhibition domain. ${ }^{22}$

\section{Screen time as a sedentary behaviour}

Sedentary behaviour was evaluated with a questionnaire. Parents were asked to report their child's average time spent on screen-based activities, such as with a computer, TV, video game, or smartphone. The screen time was reported in minutes for weekdays and weekends separately. It was calculated in terms of 30 minutes, and weekdays and weekends were combined and divided by seven days. Therefore, screen time 5 indicates 2.5 hours.

\section{Family socioeconomic status}

Information on occupational status and education of both parents was used to calculate the SES score. It was classified into five levels: lowest (unemployed and 6 years of education or less), low (lower vocational training and 9 years of education or less), medium (intermediate vocational training, 12 years of education or less), high (completed higher vocational training and 16 years of education or less), and highest (professional and more than 16 years of education). Data on other indicators for SES (job position and household income) were not available. However, the educational level of parents was known to correlate with SES overall and to be associated with parental knowledge, lifestyle, and habits. ${ }^{23}$

\section{Parental variables}

Parental smoking and drinking were reported by parents in the questionnaire using the categories 'never', 'occasionally', and 'regularly'. Responding as never or occasionally was condensed into absence of smoking or drinking for statistical analysis. For parental pathological drinking, additional data were added from the CAST as rated by participants. The CAST is a widely used 30-item inventory assessing an individual's experiences in relation to their parents' drinking behaviour. ${ }^{24}$ Scores can range from 0 to 30 ; scores of 6 or more indicate that the individual is likely the offspring of an alcoholic parent. The Ko- rean version of CAST was developed and found to be a reliable and valid measure of parental alcoholism with a Cronbach's alpha of $0.96 .^{25}$

\section{Statistical analysis}

Descriptive analyses of the full sample were conducted by calculating the distribution of demographic variables, BMI zscore, ADHD symptoms, potential mediators and confounders. To assess the relationship among a BMI z-score, ADHD symptoms, and all other variables, we calculated zero-order correlation and partial correlation statistics. We conducted hierarchical regression analysis to explore the interaction effects between potential mediators and the gender of the child on the link of ADHD symptoms with the BMI z-score. Variables used to create interaction terms were mean centred (standardized), and the cross-product terms were created using standardized values. ${ }^{26}$ We used hierarchical linear regression models of the BMI z-score and the PROCESS macro for IBM SPSS Version 22 (IBM Corp., Armonk, NY, USA) to determine whether uncontrolled eating and screen time mediate the hypothesized relationship between ADHD symptoms and BMI $\mathrm{z}$-score, adjusting for age, emotional symptoms, family SES, and parental variables. We tested the model with a bootstrapped sample of 5000 with confidence intervals (CIs) set at $95 \%$. We used the bootstrapping procedure recommended by Preacher and Hayes, in which assumptions are more realistic than in the Sobel test. ${ }^{27}$

\section{RESULTS}

\section{Description}

As shown in Table 1, the percentage of overweight including obesity was $18.3 \%$ in our data: $20.4 \%$ for boys and $16.5 \%$ for girls. SDQ-HI scores for ADHD symptoms were at-risk in $12.5 \%$ and pathological in $5.5 \%$ of the children: $7.0 \%$ for boys and $4.1 \%$ for girls.

\section{Confounders associated with ADHD symptoms and BMI z-score}

Table 2 shows a significant association between ADHD symptoms and BMI z-scores, which remained after controlling for possible confounders (gender, age, emotional symptoms, family SES, parental smoking and problematic drinking; partial $\mathrm{r}=0.24, \mathrm{p}<0.001$ ). Most confounders were associated significantly with potential mediators (uncontrolled eating and screen time), ADHD symptom scores, and BMI z-scores. We therefore adjusted for them as covariates in the regression models.

\section{Moderation by gender}

In hierarchical regression analysis with uncontrolled eating 
JS Ahn et al.

Table 1. Descriptive data of the sample $(\mathrm{N}=327)$

\begin{tabular}{|c|c|c|c|c|}
\hline Variables & All $(\mathrm{N}=327)$ & Boys $(\mathrm{N}=157)$ & Girls $(\mathrm{N}=170)$ & $\mathrm{p}$ \\
\hline Age, $y$, mean (SD) & $11.90(0.72)$ & $11.89(0.72)$ & $11.90(0.71)$ & \\
\hline BMI, mean (SD) & $18.78(2.95)$ & $19.20(3.19)$ & $18.38(2.67)$ & $<0.05$ \\
\hline BMI z-score, mean (SD) & $-0.0447(0.9856)$ & $-0.0186(1.0226)$ & $-0.0685(0.9527)$ & \\
\hline Overweight+obesity, N (\%) & $60(18.3)$ & $32(20.4)$ & $28(16.5)$ & \\
\hline ADHD symptoms, mean (SD) & $4.35(1.80)$ & $4.76(1.85)$ & $3.96(1.66)$ & $<0.001$ \\
\hline $\mathrm{ADHD}, \mathrm{N}(\%)$ & $18(5.5)$ & $11(7.0)$ & $7(4.1)$ & \\
\hline Emotional symptoms, mean (SD) & $3.26(2.17)$ & $3.18(2.07)$ & $3.33(2.27)$ & \\
\hline Uncontrolled eating, mean (SD) & $30.86(4.76)$ & $31.69(5.01)$ & $30.10(4.38)$ & $<0.01$ \\
\hline Screen time/30 min., mean (SD) & $8.06(3.45)$ & $8.48(3.69)$ & $7.68(3.18)$ & $<0.05$ \\
\hline Family SES, mean (SD) & $3.24(0.63)$ & $3.46(0.58)$ & $3.03(0.61)$ & $<0.001$ \\
\hline Parental smoking, N (\%) & $168(51.4)$ & $84(53.5)$ & $84(49.4)$ & \\
\hline Parental problematic drinking, N (\%) & $152(46.5)$ & $80(51.0)$ & $72(42.4)$ & \\
\hline
\end{tabular}

ADHD: SDQ-HI score $\geq 8$. Family SES: $1=$ highest, $5=$ lowest. Parental problematic drinking: The CAST score $\geq 6$. BMI: body mass index, ADHD: attention deficit hyperactivity disorder, SDQ-HI: Strengths and Difficulties Questionnaire-hyperactivity inattention scale, CAST: children of alcoholics screening test

Table 2. Zero-order correlation matrix for all variables $(\mathrm{N}=327)$

\begin{tabular}{|c|c|c|c|c|c|c|c|c|c|}
\hline Variables & 1 & 2 & 3 & 4 & 5 & 6 & 7 & 8 & 9 \\
\hline 1. Gender & 1 & & & & & & & & \\
\hline 2. Age & -0.008 & & & & & & & & \\
\hline 3. BMI z-score & 0.025 & -0.048 & & & & & & & \\
\hline 4. ADHD symptoms & $0.221^{\dagger}$ & 0.037 & $0.280^{\dagger}$ & & & & & & \\
\hline 5. Uncontrolled eating & $0.167^{\dagger}$ & $0.148^{\dagger}$ & $0.322^{\dagger}$ & $0.468^{\dagger}$ & & & & & \\
\hline 6. Screen time & $0.116^{*}$ & 0.020 & $0.129^{*}$ & $0.318^{\dagger}$ & $0.353^{\dagger}$ & & & & \\
\hline 7. Emotional symptoms & -0.035 & -0.028 & 0.091 & $0.185^{\dagger}$ & 0.101 & $0.137^{*}$ & & & \\
\hline 8. Family SES & $0.343^{\dagger}$ & -0.093 & $0.236^{\dagger}$ & $0.202^{\dagger}$ & $0.138^{*}$ & $0.162^{\dagger}$ & 0.076 & & \\
\hline 9. Parental smoking & 0.041 & -0.089 & 0.055 & 0.085 & 0.065 & $-0.124^{*}$ & -0.065 & 0.086 & \\
\hline 10. Parental problematic drinking & 0.086 & 0.057 & 0.091 & $0.189^{\dagger}$ & $0.138^{*}$ & $0.109^{*}$ & $0.181^{\dagger}$ & 0.094 & $0.146^{\dagger}$ \\
\hline
\end{tabular}

Male gender, presence of parental smoking, and parental problematic drinking were transformed to dummy variable, 1 . Family SES: $1=$ highest, $5=$ lowest. ${ }^{*} \mathrm{p}<0.05,{ }^{\dagger} \mathrm{p}<0.01$. BMI: body mass index, ADHD: attention deficit hyperactivity disorder

Table 3. Gender as a moderator in the mediation model $(\mathrm{N}=327)$

\begin{tabular}{|c|c|c|c|c|c|c|}
\hline Dependent variable & Model & Predictor & $\mathrm{R}^{2}$ & $\Delta \mathrm{R}^{2}$ & $\mathrm{~F}$ & $\mathrm{p}$ \\
\hline \multirow[t]{3}{*}{ Uncontrolled eating } & 1 & ADHD symptoms & 0.219 & 0.219 & 90.929 & $<0.001$ \\
\hline & 2 & ADHD symptoms, Gender & 0.223 & 0.004 & 46.468 & $<0.001$ \\
\hline & 3 & ADHD symptoms, Gender, ADHD symptoms $\times$ Gender & 0.225 & 0.002 & 31.217 & $<0.001$ \\
\hline \multirow[t]{3}{*}{ BMI z-score } & 1 & UE & 0.103 & 0.103 & 37.466 & $<0.001$ \\
\hline & 2 & UE, Gender & 0.104 & 0.001 & 18.842 & $<0.001$ \\
\hline & 3 & UE, Gender, UE $\times$ Gender & 0.105 & 0.001 & 12.660 & $<0.001$ \\
\hline \multirow[t]{3}{*}{ Screen time } & 1 & ADHD symptoms & 0.101 & 0.101 & 36.558 & $<0.001$ \\
\hline & 2 & ADHD symptoms, Gender & 0.103 & 0.002 & 18.670 & $<0.001$ \\
\hline & 3 & ADHD symptoms, Gender, ADHD symptoms $\times$ Gender & 0.106 & 0.003 & 12.831 & $<0.001$ \\
\hline \multirow[t]{3}{*}{ BMI z-score } & 1 & Screen time & 0.017 & 0.017 & 5.491 & $<0.01$ \\
\hline & 2 & Screen time, Gender & 0.017 & 0.000 & 2.755 & n.s. \\
\hline & 3 & Screen time, Gender, Screen time $\times$ Gender & 0.025 & 0.008 & 2.716 & $<0.01$ \\
\hline
\end{tabular}

All continuous variables were mean centred, and the models were controlled for age, emotional symptoms, family SES, parental smoking, and parental problematic drinking. BMI: body mass index, UE: uncontrolled eating, ADHD: attention deficit hyperactivity disorder, n.s.: not statistically significant 
and screen time (mediators) as dependent variables, ADHD symptoms, gender (moderator), and $\mathrm{ADHD}$ symptoms $\times$ gender interaction increased $\mathrm{R}^{2}$ (accountability) on the mediators significantly, as shown in Table 3. In the second regression, with the BMI z-score as a dependent variable, the mediators gender and mediator $\times$ gender interaction increased $\mathrm{R}^{2}$ on the BMI z-score significantly. To confirm the interaction effects of gender, a separate analysis was performed for each gender.

\section{Mediation by uncontrolled eating and screen time}

With hierarchical regression analysis, we examined whether the relationship between ADHD symptoms and BMI zscores were mediated by uncontrolled eating and screen time.
Table 4 presents for boys and girls the unstandardized regression coefficients (B) with standard errors (SE) and confidence intervals estimating uncontrolled eating and screen time (mediators) and BMI z-scores (controlling for age, emotional symptoms, family SES, and parental smoking and problematic drinking). ADHD symptoms were significantly associated with two mediators and with BMI z-scores for both genders. For boys, when ADHD symptoms and two mediators were included in the model, the association of uncontrolled eating with BMI $\mathrm{z}$-scores remained significant, whereas the association of ADHD symptoms and screen time with BMI $\mathrm{z}$-scores did not. Thus, for boys, uncontrolled eating acted as a complete mediator. For girls, when ADHD symptoms and

Table 4. Hierarchical linear regression model of BMI z-score in boys and girls

\begin{tabular}{|c|c|c|c|c|c|c|c|c|}
\hline \multirow{2}{*}{ Level } & \multirow{2}{*}{ Dependent variable } & \multirow{2}{*}{ Predictor } & \multirow{2}{*}{$\mathrm{R}^{2}$} & \multicolumn{2}{|c|}{ Unstandardized coefficients } & \multirow{2}{*}{$\mathrm{t}$} & \multirow{2}{*}{$\mathrm{p}$} & \multirow{2}{*}{$95 \% \mathrm{CI}$} \\
\hline & & & & B & SE & & & \\
\hline \multicolumn{9}{|c|}{ Boys $(\mathrm{N}=157)$} \\
\hline & BMI z-score & ADHD symptoms & $0.1684^{\ddagger}$ & 0.0991 & 0.0438 & 2.2627 & $<0.05$ & $0.0126-0.1856$ \\
\hline & UE & ADHD symptoms & $0.2749^{\ddagger}$ & 1.2368 & 0.2005 & 6.1676 & $<0.001$ & $0.8406-1.6331$ \\
\hline & Screen time & ADHD symptoms & 0.0668 & 0.4550 & 0.1672 & 2.7208 & $<0.01$ & $0.1246-0.7855$ \\
\hline & BMI z-score & UE & $0.2463^{\ddagger}$ & 0.0682 & 0.0175 & 3.8913 & $<0.001$ & $0.0336-0.1028$ \\
\hline & & Screen time & & -0.0258 & 0.0210 & -1.2263 & n.s. & $-0.0673-0.0158$ \\
\hline & & ADHD symptoms & & 0.0265 & 0.0472 & 0.5607 & n.s. & $-0.0669-0.1199$ \\
\hline \multicolumn{9}{|c|}{ Girls (N=170) } \\
\hline & BMI z-score & ADHD symptoms & $0.1173^{\dagger}$ & 0.1638 & 0.0403 & 3.8122 & $<0.001$ & $0.0789-0.2486$ \\
\hline & UE & ADHD symptoms & $0.2696^{\ddagger}$ & 1.0198 & 0.1798 & 5.6711 & $<0.001$ & $0.6647-1.3748$ \\
\hline & Screen time & ADHD symptoms & $0.2050^{\ddagger}$ & 0.5985 & 0.1381 & 4.3976 & $<0.001$ & $0.3298-0.8673$ \\
\hline & BMI z-score & UE & $0.1529^{\dagger}$ & 0.0393 & 0.0191 & 2.0607 & $<0.05$ & $0.0016-0.0770$ \\
\hline & & Screen time & & 0.0253 & 0.0252 & 1.0052 & n.s. & $-0.0244-0.0751$ \\
\hline & & ADHD symptoms & & 0.1085 & 0.0474 & 2.2879 & $<0.05$ & $0.0148-0.2022$ \\
\hline
\end{tabular}

All controlled for age, emotional symptoms, family SES, parental smoking, and parental problematic drinking. BMI: body mass index, UE: uncontrolled eating, n.s.: not statistically significant, ADHD: attention deficit hyperactivity disorder

Table 5. Gender-specific outcomes of bootstrapping technique: indirect effects of screen time and uncontrolled eating in the mediation between ADHD symptoms and BMI Z-score

\begin{tabular}{|c|c|c|c|c|}
\hline \multirow{2}{*}{ Route of indirect effects } & \multirow{2}{*}{$\begin{array}{c}\text { Effect } \\
\text { coefficient }\end{array}$} & \multirow{2}{*}{ Boot SE } & \multicolumn{2}{|c|}{$95 \% \mathrm{CI}$} \\
\hline & & & Boot LLCI & Boot ULCI \\
\hline \multicolumn{5}{|l|}{ Boys $(\mathrm{N}=157)$ : direct effect $\mathrm{t}=0.5607, \mathrm{p}=\mathrm{n} . \mathrm{s}$. } \\
\hline ADHD symptoms $\rightarrow$ uncontrolled eating $\rightarrow$ BMI z-score & 0.0843 & 0.0229 & 0.0457 & 0.1365 \\
\hline ADHD symptoms $\rightarrow$ screen time $\rightarrow$ BMI $z$-score & -0.0117 & 0.0115 & -0.0424 & 0.0047 \\
\hline Overall indirect effects & 0.0726 & 0.0282 & 0.0360 & 0.1289 \\
\hline \multicolumn{5}{|l|}{ Girls $(\mathrm{N}=170)$ : direct effect $\mathrm{t}=2.2879, \mathrm{p}<0.05$} \\
\hline ADHD symptoms $\rightarrow$ uncontrolled eating $\rightarrow$ BMI z-score & 0.0401 & 0.0202 & 0.0063 & 0.0873 \\
\hline ADHD symptoms $\rightarrow$ screen time $\rightarrow$ BMI z-score & 0.0152 & 0.0142 & -0.0097 & 0.0469 \\
\hline Overall indirect effects & 0.0553 & 0.0226 & 0.0139 & 0.1038 \\
\hline
\end{tabular}

All controlled for age, emotional symptoms, family SES, parental smoking, and parental problematic drinking. Boot SE: Bootstrapping standard error, LLCI: lower limit of the confidence interval, ULCI: upper limit of the confidence interval, n.s.: not statistically significant, CI: confidence interval, ADHD: attention deficit hyperactivity disorder 


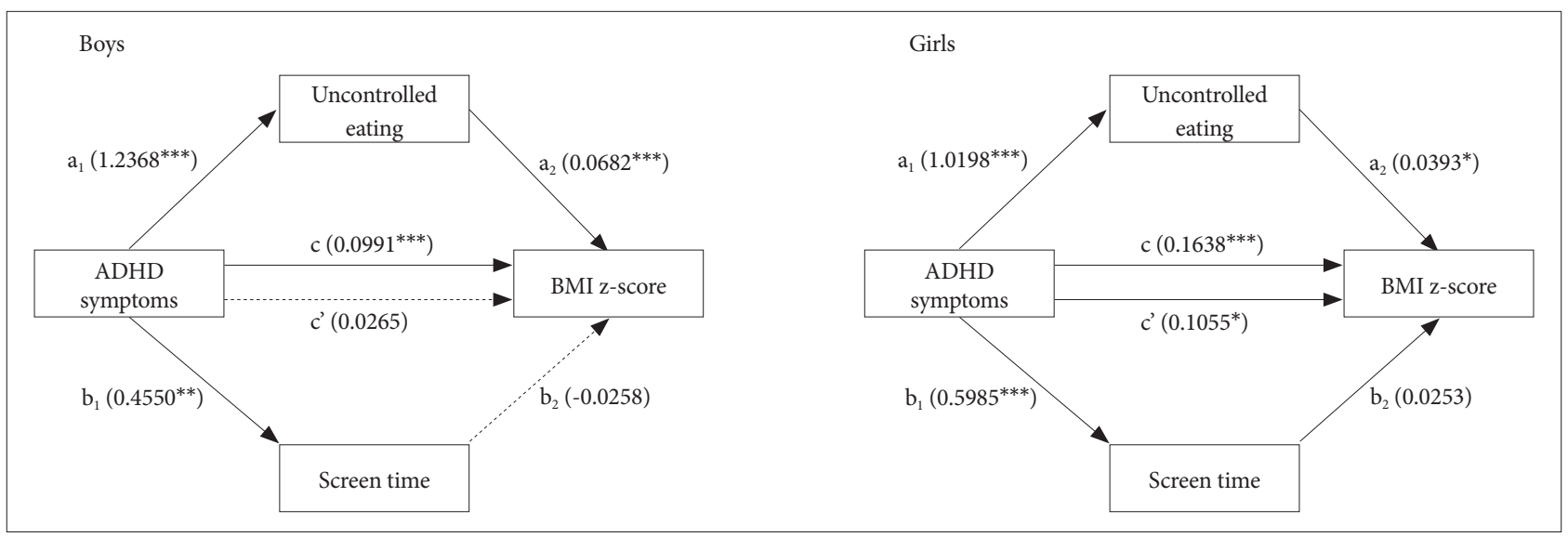

Figure 1. Gender-specific outcome of the tested model. c=Total effect of ADHD symptoms on BMI z-score: $c^{\prime}+\left[\left(a_{1} \times a_{2}\right)+\left(b_{1} \times b_{2}\right)\right] . c^{\prime}=$ Direct effect of ADHD symptoms on BMl z-score, independent of screen time and uncontrolled eating. All controlled for age, emotional symptoms, family SES, parental smoking and parental problematic drinking. ${ }^{*} p<0.05,{ }^{* *} p<0.01,{ }^{* * *} p<0.001$. ADHD: attention deficit hyperactivity disorder, BMI: body mass index.

two mediators were included in the model, the association of $\mathrm{ADHD}$ symptoms and uncontrolled eating with the BMI zscore remained significant, whereas the association of screen time with BMI z-scores did not. These results indicate that uncontrolled eating acted as a partial mediator in girls.

\section{Bootstrapping test for indirect effect through mediating paths}

We performed a bootstrapping test to verify whether the mediating effects of uncontrolled eating and screen time were statistically significant in the indirect impact of ADHD symptoms on BMI z-scores. For boys, the increase of the ADHD symptoms had a positive impact on uncontrolled eating, and the mediating path for higher uncontrolled eating that in turn increased BMI z-scores was statistically significant. Whereas the increase of ADHD symptoms had a positive impact on screen time, the mediating path of the higher screen time in turn decreased BMI z-scores in boys, reducing the overall indirect effect of the mediating path. For girls, although uncontrolled eating only was verified as a mediator with statistical significance, both mediating paths had a positive impact on BMI z-scores.

Results as shown in Table 4 and 5 indicate that there was an indirect effect of ADHD symptoms on BMI z-scores completely mediated by uncontrolled eating for boys. However, ADHD symptoms had stronger direct impact on BMI z-scores for girls. Figure 1 shows hypothesized path models with path coefficients for boys and girls separately. We calculated a total effect coefficient (c) by the sum of the direct effect coefficient ( $c^{\prime}$ ) and indirect effect coefficient $\left[\left(a_{1} \times a_{2}\right)+\left(b_{1} \times b_{2}\right)\right]$.

\section{DISCUSSION}

Numerous studies on the association between childhood
$\mathrm{ADHD}$ and BMI in adults and adolescents have been performed, but a clear understanding of the behavioural variables that contribute to the association has yet to be established. Moreover, to our knowledge, a gender-specific mediation model with behavioural variables for children has never been tested. Thus, we are the first to report that the link of ADHD symptoms with BMI z-score among children is mediated by uncontrolled eating behaviour, which is stronger in boys than in girls. Controlling major confounders, we were able not only to confirm the ADHD symptoms-BMI link, but also to address the issue of behavioural mediators and gender difference.

Regarding the mediation analysis, we found that for boys there was no direct effect of ADHD symptoms on BMI. Some researchers have argued that the relationship between ADHD and overweight depends on the main symptoms of ADHD. Through the interview of young adults, Cortese et al. ${ }^{28}$ found that impulsive and inattentive symptoms, but not hyperactive symptoms, mediated the association. Similarly, a Chinese study of children aged 6-16 years found that youths with the combined subtype of ADHD were significantly more likely to be overweight compared to those with only inattentive or only hyperactive symptoms. ${ }^{29}$ We assessed the ADHD symptoms with the SDQ-HI scale and could not measure the three core symptoms separately. Boys with ADHD may be more likely to be hyperactive than girls, which may be a plausible explanation for the gender difference in our data. ${ }^{30}$

Although many questions concerning eating behaviours associated with overweight still remain unanswered, it is possible that people with ADHD are characterized by behavioural dysregulation as well as delay aversion, which could foster the development of eating patterns that lead to an increased risk for obesity. ${ }^{31}$ A study on adults with childhood episodes of ADHD found that binge eating, but not depression, partially mediated the association. ${ }^{32}$ Our study results for girls were in 
line with the findings in that uncontrolled eating acted as a partial mediator and that emotional symptoms were not correlated with BMI.

A novel finding in our data was the gender specificity; the mediating effect of uncontrolled eating on the ADHD symptoms-BMI link was stronger in boys than in girls. We note that Canadian research on the eating behaviour traits associated with BMI in adolescents showed similar results. Using the TFEQ, Gallant et al. ${ }^{33}$ found a positive relationship between uncontrolled eating and BMI only in boys. Because boys were less focused on than girls in studies on eating behaviours, it is difficult to explain the gender difference found in the present study. However, it has been shown that uncontrolled eating combined with high dietary restraint made girls more likely to fail in restraining themselves and to binge. ${ }^{34}$ We can speculate that possibly for this reason, uncontrolled eating acted as a partial mediator among girls. Unfortunately, the assessment of dietary restraint was not included in the present study. Another speculation comes from the study reporting that eating-disorder symptoms generally increase from age 14 in girls, whereas boys may show reductions in eating pathology from early adolescence. ${ }^{35}$ Accordingly, our findings may be reasonable for the preadolescent age of participants.

In our data, screen time had no mediating effect on the ADHD symptoms-BMI link for boys but had a small effect without statistical significance for girls. Kim et al..$^{14}$ found that girls with ADHD were more likely than healthy peers to exceed recommended levels of sedentary behaviour, but boys with $\mathrm{ADHD}$ were not. A recent meta-analysis presented strong evidence for a relationship of screen time with BMI among healthy children, but no evidence was found for the relationship when total sedentary time was assessed objectively. ${ }^{35}$ Likewise, a longitudinal study using an accelerometer to measure sedentary activity of children in the sixth and eighth grades among girls showed that the increase in sedentary activity was not associated with BMI. ${ }^{36}$ Meanwhile, it has been suggested that the relationship depends on the type of sedentary behaviour as well as gender. Regarding TV viewing time, strong evidence for a positive relationship with overweight was found, but there was no evidence of a relationship with computer use/ game time ${ }^{35}$ For girls, overweight was correlated positively with TV viewing, but in contrast, there was no association for boys. ${ }^{37}$ We did not separate out different types of sedentary behaviour and measured screen time by parent-report, as opposed to a TV/computer monitoring device or observations. Considering that children with ADHD are more addicted to Internet games, the use of electronic media should be assessed more specifically in future studies. ${ }^{38}$

Of all confounders assessed in the present study, the emotional symptoms seem notable. Even though the reciprocal as- sociation between depression and overweight among youths is theoretically appealing, a cross-sectional study reported that the correlations were 0.08 for girls and -0.01 for boys, ${ }^{39}$ which is consistent with our data (partial $r=0.02, p=0.722$ ). However, Pauli-Pott et al. ${ }^{12}$ suggested that anxiety or depression would be necessary for overweight youths with ADHD to develop binge eating. Interestingly, longitudinal studies found that the unfavourable effect of depression and overweight on each other may be reinforced by time. ${ }^{40,41}$

Our additional finding in regard to the confounders is that parental problematic drinking is associated significantly with ADHD symptoms, uncontrolled eating, and screen time. Addiction and eating behaviour are thought to be regulated by the dopamine circuit in the brain like ADHD symptoms. According to one study, children of alcoholics (COAs) aged 15-18 spent more time in sedentary behaviour, had poorer eating habits, higher substance use, and more emotional symptoms on the SDQ. ${ }^{42}$ These findings are in line with our results except for a lack of association with ADHD symptoms, which may be because of the age-relatedness of ADHD and substance use disorder diagnosis.

Although our primary focus is on evaluating the mediators and gender difference in the ADHD-weight link, further exploring the two confounders in the future study on older adolescents is warranted to obtain a better understanding of the association between ADHD symptoms and BMI.

\section{Limitations and strengths}

Our findings have some limitations and should be interpreted with caution. First, the assessment of ADHD symptoms was based on parent reporting, which is likely to be biased although the prevalence of ADHD on the basis of the cut-off point in our data was similar to the expected estimation of the community sample. Second, we assessed screen time as a variable of sedentary behaviour, and it was based on parent reporting. Total sedentary time should be examined with a combination of various screen-based and non-screen-based activities and measured objectively for the clarity of its mediating role. Furthermore, considering that one study result showed that adolescents with daily screen time $>5$ hours had increased risk of abdominal obesity, further studies may focus on the subjects with serious sedentary behaviour problems. ${ }^{43}$ Third, we used a dimensional approach for data analysis of ADHD symptoms and BMI instead of categorical diagnosis. However, binary classification for continuous variables may not fully explain the nature of both conditions. In other words, there is potential for misclassification that could lead to a reduced reliability of the observed relationships. Finally, although we tested mediators on the link between ADHD and weight, a causality or temporal relationship could not be established in 
this cross-sectional study. In addition, our results should be replicated in the subjects with obesity or overweight.

Strengths of the present study include the narrow age band that minimises an age-dependent effect, the simultaneous investigation of several important confounders including emotional symptoms and parental problematic drinking, objective measuring of weight and height by professionals, and gender comparison. Additionally, given that most research on this issue has been conducted in Western countries, findings of the study on Korean preadolescents may be informative for future research on cross-cultural differences.

\section{Conclusion}

We conducted the present study to examine the link of ADHD symptoms with overweight in late childhood and to assess the gender-specific role of uncontrolled eating and screen time as mediators of the link. Although the accountability was small, our results showed that the association was evident and mediated by uncontrolled eating, not by screen time, completely among boys and partially among girls. Considering that late childhood is a particularly important period for age- and gender-specific conditions of $\mathrm{ADHD}$, eating disorders, and overweight, our study findings could help to tailor intervention programs to meet the specific needs of this subgroup.

\section{REFERENCES}

1. World Health Organization. Childhood overweight and obesity.http:// www.who.int/dietphysicalactivity/childhood/en/. Accessed January 5, 2017.

2. Cortese S, Moreira-Maia CR, St Fleur D, Morcillo-Penalver C, Rohde LA, Faraone SV. Association between ADHD and obesity: a systematic review and meta-analysis. Am J Psychiatry 2016;173:34-43.

3. Choudhry Z, Sengupta SM, Grizenko N, Thakur GA, Fortier ME, Schmitz N, et al. Association between obesity-related gene FTO and ADHD. Obesity (Silver Spring) 2013;21:E738-E744.

4. Seymour KE, Reinblatt SP, Benson L, Carnell S. Overlapping neurobehavioral circuits in ADHD, obesity, and binge eating: evidence from neuroimaging research. CNS Spectr 2015;20:401-411.

5. Cortese S, Castellanos FX. The relationship between ADHD and obesity: implications for therapy. Expert Rev Neurother 2014;14:473-479.

6. Erhart M, Herpertz-Dahlmann B, Wille N, Sawitzky-Rose B, Holling H, Ravens-Sieberer U. Examining the relationship between attention-deficit/hyperactivity disorder and overweight in children and adolescents. Eur Child Adolesc Psychiatry 2012;21:39-49.

7. Nederkoorn C, Jansen E, Mulkens S, Jansen A. Impulsivity predicts treatment outcome in obese children. Behav Res Ther 2007;45:10711075.

8. Davis C, Levitan RD, Smith M, Tweed S, Curtis C. Associations among overeating, overweight, and attention deficit/hyperactivity disorder: a structural equation modelling approach. Eat Behav 2006;7:266-274.

9. Tremblay MS, LeBlanc AG, Kho ME, Saunders TJ, Larouche R, Colley $\mathrm{RC}$, et al. Systematic review of sedentary behaviour and health indicators in school-aged children and youth. Int J Behav Nutr Phy Act 2011; 8:98.

10. Waring ME, Lapane KL. Overweight in children and adolescents in relation to attention-deficit/hyperactivity disorder: results from a national sample. Pediatrics 2008;122:e1-e6.
11. Lingineni RK, Biswas S, Ahmad N, Jackson BE, Bae S, Singh KP. Factors associated with attention deficit/hyperactivity disorder among US children: results from a national survey. Bmc Pediatr 2012;12:50.

12. Pauli-Pott U, Becker K, Albayrak O, Hebebrand J, Pott W. Links between psychopathological symptoms and disordered eating behaviors in overweight/obese youths. Int J Eat Disorder 2013;46:156-163.

13. Wilens TE, Biederman J, Brown S, Tanguay S, Monuteaux MC, Blake C, et al. Psychiatric comorbidity and functioning in clinically referred preschool children and school-age youths with ADHD. J Am Acad Child Adolesc Psychiatry 2002;41:262-268.

14. Kim J, Mutyala B, Agiovlasitis S, Fernhall B. Health behaviors and obesity among US children with attention deficit hyperactivity disorder by gender and medication use. Prev Med 2011;52:218-222.

15. van Egmond-Frohlich AW, Widhalm K, de Zwaan M. Association of symptoms of attention-deficit/hyperactivity disorder with childhood overweight adjusted for confounding parental variables. Int J Obes (London) 2012;36:963-968.

16. Kelder SH, Perry CL, Klepp KI, Lytle LL. Longitudinal tracking of adolescent smoking, physical activity, and food choice behaviors. Am J Public Health 1994;84:1121-1126.

17. Goodman R. The strengths and difficulties questionnaire: a research note. J Child Psychol Psychiatry 1997;38:581-586.

18. Ahn J, Jun S, Han J, Noh K, Goodman R. The development of a Korean version of the Strengths and Difficulties Questionnaire. J Korean Neuropsychiatr Assoc 2003;42:141-147.

19. Korea Centers for Disease Control and Prevention, The Korean Pediatric Society, The Committee for Korean Children and Adolescents Growth Standard. 2007 Korean Children and Adolescents Growth Standard (commentary for the development of 2007 growth chart). Seoul: Division of Chronic Disease Surveillance in Korea Centers for Disease Control and Prevention; 2007.

20. Stunkard AJ, Messick S. The three-factor eating questionnaire to measure dietary restraint, disinhibition and hunger. J Psychosom Res 1985; 29:71-83.

21. Arnow B, Kenardy J, Agras WS. The emotional eating scale: the development of a measure to assess coping with negative affect by eating. Int J Eat Disord 1995;18:79-90.

22. Kim M, Kim S. The three dimensions of eating behavior: development of scale for assessing cognitive restraint of eating, disinhibition, and hunger. J Korean Nurs Assoc 1996;27:377-388.

23. Bornstein M, Bradley R. Socioeconomic Status, Parenting, and Child Development. Mahwah, NJ: Lawrence Erlbaum Associates; 2003.

24. Jones J. Children of Alcoholics Screening Test (CAST). Chicago: Camelot; 1983.

25. Kim M, Chang H, Kim K. Development of the Korean version of the children of alcoholics screening test (CAST-K): a reliability and validity study. J Korean Neuropsychiatr Assoc 1995;34:1182-1193.

26. Cohen J, Cohen P, West SH, Aiken LS. Applied Multiple Regression/ Correlation Analysis for the Behavioral Sciences. Mahwah, NJ: Lawrence Erlbaum Associates; 2003.

27. Preacher KJ, Hayes AF. Asymptotic and resampling strategies for assessing and comparing indirect effects in multiple mediator models. Behav Res Methods 2008;40:879-891.

28. Cortese S, Faraone SV, Bernardi S, Wang S, Blanco C. Adult attentiondeficit hyperactivity disorder and obesity: epidemiological study. $\mathrm{Br} \mathrm{J}$ Psychiatry 2013;203:24-34.

29. Yang RW, Mao SJ, Zhang SH, Li R, Zhao ZY. Prevalence of obesity and overweight among Chinese children with attention deficit hyperactivity disorder: a survey in Zhejiang Province, China. BMC Psychiatry 2013; 13:133.

30. Rucklidge JJ. Gender differences in attention-deficit/hyperactivity disorder. Psychiatr Clin North Am 2010;33:357-373.

31. Davis C. Attention-deficit/hyperactivity disorder: associations with overeating and obesity. Curr Psychiatry Rep 2010;12:389-395.

32. Pagoto SL, Curtin C, Lemon SC, Bandini LG, Schneider KL, Bodenlos 
JS, et al. Association between adult attention deficit/hyperactivity disorder and obesity in the US population. Obesity (Silver Spring) 2009;17: 539-544.

33. Gallant AR, Tremblay A, Perusse L, Bouchard C, Despres JP, Drapeau $\mathrm{V}$. The three-factor eating questionnaire and BMI in adolescents: results from the Quebec Family Study. Brit J Nutr 2010;104:1074-1079.

34. van Strien $T$. The concurrent validity of a classification of dieters with low versus high susceptibility toward failure of restraint. Addict Behav 1997;22:587-597.

35. van Ekris E, Altenburg TM, Singh AS, Proper KI, Heymans MW, Chinapaw MJ. An evidence-update on the prospective relationship between childhood sedentary behaviour and biomedical health indicators: a systematic review and meta-analysis. Obes Rev 2016;17:833-849.

36. Treuth MS, Baggett CD, Pratt CA, Going SB, Elder JP, Charneco EY, et al. A longitudinal study of sedentary behavior and overweight in adolescent girls. Obesity (Silver Spring) 2009;17:1003-1008.

37. Dupuy M, Godeau E, Vignes C, Ahluwalia N. Socio-demographic and lifestyle factors associated with overweight in a representative sample of 11-15 year olds in France: results from the WHO-Collaborative Health Behaviour in School-aged Children (HBSC) cross-sectional study. BMC
Public Health 2011;11:442.

38. Ha JH, Yoo HJ, Cho IH, Chin B, Shin D, Kim JH. Psychiatric comorbidity assessed in Korean children and adolescents who screen positive for Internet addiction. J Clin Psychiatry 2006;67:821-826.

39. Needham BL, Crosnoe R. Overweight status and depressive symptoms during adolescence. J Adolescent Health 2005;36:48-55.

40. Stice E, Presnell K, Spangler D. Risk factors for binge eating onset in adolescent girls: a 2-year prospective investigation. Health Psychol 2002;21:131-138.

41. Luppino FS, de Wit LM, Bouvy PF, Stijnen T, Cuijpers P, Penninx BW, et al. Overweight, obesity, and depression a systematic review and meta-analysis of longitudinal studies. Arch Gen Psychiatry 2010;67:220229.

42. Serec M, Svab I, Kolsek M, Svab V, Moesgen D, Klein M. Health-related lifestyle, physical and mental health in children of alcoholic parents. Drug Alcohol Rev 2012;31:861-870.

43. Chung WC, Cho YG, Kang JH, Park HA, Kim KW, Kang JH, et al. Lifestyle habits related to abdominal obesity in Korean adolescents. Korean J Fam Med 2010;31:547-554. 